\title{
Self-sensing Hybrid Composite Rod with Braided Reinforcement for Structural Health Monitoring
}

\author{
K. P. Rosado ${ }^{1, a}$, Sohel Rana ${ }^{1, b}$, C. Pereira ${ }^{2, c}$ and R. Fangueiro ${ }^{1, d}$ \\ ${ }^{1}$ Fibrous Materials Research Group, Department of Engineering Textile, University of Minho, \\ Guimaraes, Portugal \\ ${ }^{2}$ Territory, Environment and Construction Centre, Civil Engineering Department, University of \\ Minho, Portugal \\ arosadomk@gmail.com, ${ }^{b}$ sohel_iitd@yahoo.com, c cristiana.pereira@civil.uminho.pt, \\ drfang@det.uminho.pt
}

Keywords: Self-monitoring; braided composites; chopped carbon fibres, strain sensitivity

\begin{abstract}
Enhancing the performance and lightness of different structures has already been achieved by the employment of fibre reinforced composite materials. Nowadays, a new challenging perspective is being given to these materials by the inclusion of non-metallic conductive components. This emerging technology will lead to multifunctional composites with possible applications in structural health monitoring and traffic monitoring. The aim is to avoid corrosion problems from metallic components, as well as to eliminate the need of expensive equipments used for the health monitoring of large infrastructures.

In the present research, the strain-sensing capability of a core-reinforced hybrid carbon fibre/glass fibre braided composite has been investigated in order to develop continuous monitoring system. The characterization of sensing behaviour was performed with the help of an instrumental set-up capable of measuring the change in electrical resistance with mechanical stresses applied to the samples. The effect of core composition (carbon fibre/glass fibre weight ratio) on the strain sensitivity of the braided composites has been studied in order to find out the optimum composition for best sensing capability. Among the three compositions studied (23/77, 47/53 and 100/0), composites with lowest amount of carbon fibre showed the best strain sensitivity with gauge factors up to 23.4 at very low flexural strain $(0.55 \%)$. Attempts have also been made in this research to develop a piezoresistive matrix for the braided composites in order to further enhance their strain sensitivity. For this purpose, the strain sensing capability of an unsaturated polyester matrix dispersed with chopped carbon fibres ( $1 \mathrm{~mm}$ and $3 \mathrm{~mm}$ lengths) at various weight $\%(0.5,0.75$ and $1.25 \%$ ) was evaluated in order to find out their optimum length and concentration. It was observed that chopped fibres with different lengths showed similar strain sensitivity, which however, improves with the decrease in their concentrations.
\end{abstract}

\section{Introduction}

Efforts have been directed in recent times towards designing smart structures with self-monitoring capability of health and damage in order to avoid accidents from sudden collapse. Fibre reinforced composites are the ideal reinforcements for such structures due to the possibility of imparting many functionalities to them by selecting different fibre/matrix systems or by tailoring their designs. Polymer matrix composites containing a conductive element such as carbon fibre were found to change their electrical resistance with deformation and damage and therefore, carbon fibre composites have been investigated widely for developing self-monitoring structures. Use of hybrid composites such as glass/carbon, glass/aramid etc. proved helpful to introduce "pseudo-ductility" and to generate alarm signal well before the structural collapse [1,2]. However, less sensitivity at low strain level has restricted their applications for developing continuous monitoring system [3]. Hence, attempts have been made by researchers to improve the sensing ability of hybrid composites at low strain using pre-stressing conditions [3], which, however, is a suitable condition for civil 
engineering structures but may not be favourable for other applications. Hybrid composites with good sensitivity have also been developed using carbon nanotubes [4]. However, dispersion of carbon nanotubes in polymeric matrices is a critical issue since improper nanotube dispersion can adversely affect the mechanical properties of composites.

The present research demonstrates the possibility of designing continuous monitoring system based on core reinforced hybrid carbon fibre/glass fibre braided composites. Hybrid composites were developed using three compositions of core with different carbon fibre/glass fibre weight ratio in order to find out the optimum composition in terms of strain sensitivity. The sensing behaviour of braided composites was characterized in cyclic flexural loading at very low strain level $(0.55 \%)$ to investigate their performance in monitoring low strain in continuous manner. Moreover, this research also demonstrated the possibility of enhancing the sensing performance of braided composites using a piezoresistive matrix dispersed with chopped carbon fibres of different lengths $(1 \mathrm{~mm}$ and $3 \mathrm{~mm})$ and at different weight $\%(0.5,0.75$ and $1.25 \%)$. The strain sensing behaviour of the chopped fibre dispersed matrix was evaluated in both monotonic and cyclic compressive loading.

\section{Experimental}

Raw materials. The details of raw materials used in this study are provided in Table 1. Chopped carbon fibres were produced by cutting continuous carbon fibres in $1 \mathrm{~mm}$ and $3 \mathrm{~mm}$ lengths.

Table 1: Details of raw materials

\begin{tabular}{|c|c|c|}
\hline Material & Manufacturer & Properties \\
\hline Continuous carbon fibre & TohoTenax & Elastic modulus $240[\mathrm{GPa}]$, tensile \\
& & strength 4.3 [GPa], strain at break \\
& & $1.8 \%$, resistivity $1,6 \times 10^{-3}[\Omega . c m]$ \\
\hline Glass fibre & Saint Gobain Vetrotex & $\begin{array}{c}\text { Elastic modulus } 73.5[\mathrm{GPa}], \text { tensile } \\
\text { strength 3.5 [GPa], strain at break } \\
\end{array}$ \\
& & $4.8 \%$ \\
\hline
\end{tabular}

Experimental methods. The braided composite rods (BCRs) were produced using polyester fibres for braided structure and a combination of glass and carbon fibres as the core reinforcement. The braiding of polyester fibres and impregnation of core fibres with polyester resin/hardener mixture were done simultaneously in a single process using a vertical braiding machine with an incorporated impregnation system, developed by authors. The composite rods were then cured at environmental temperature and moisture conditions $\left(20 \pm 2^{\circ} \mathrm{C}\right.$ and $\left.50 \pm 5 \%\right)$. Chopped carbon fibres were dispersed in the polyester matrix using manual mixing with a glass rod and subsequently cured at environmental temperature for 12 hours followed by a post curing at $70^{\circ} \mathrm{C}$ for 3 hours. The distribution of chopped fibres within the matrix was analyzed with the help of optical microscopy. For this purpose, thin sections were cut from different area of the samples and analyzed in the reflectance mode of an optical microscope.

Characterization of piezoresistivity. The strain sensing behaviour of the braided composite rods was characterized by measuring the change in electrical resistance between the sample ends using two terminal dc method under cyclic 3-point flexural loading. The electrical resistance of the samples was continuously measured during the flexural test by making electrical connections between the two probes of a digital multi-meter (Agilent 84401A) and sample ends through gold wires fixed to the samples using silver paste. Similarly, both faces of the chopped fibre dispersed samples were painted with silver paste in order to ensure good electrical contacts during the compression tests. The experimental setup for piezoresistive characterization is shown in Fig. 1. 


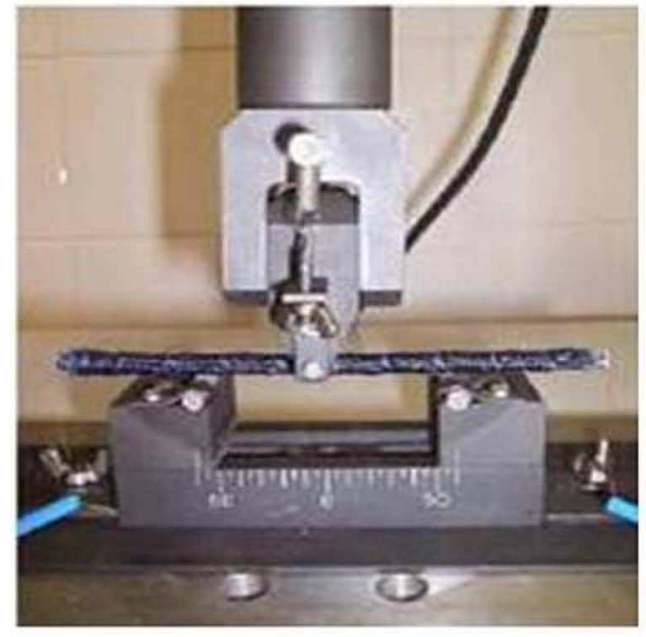

(a)

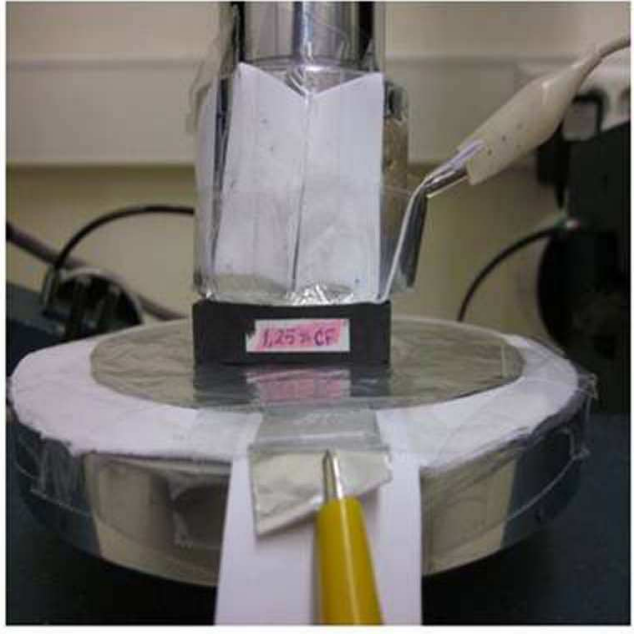

(b)

Fig. 1: Set up for piezoresistive characterization of braided composites (a) and chopped carbon fibre dispersed matrix (b)

The strain sensing capability of the composites was evaluated in terms of gauge factor (GF), which is defined in Eq. 1:

$$
G F=\frac{\frac{\Delta R}{R}}{\varepsilon}
$$

where, $\Delta \mathrm{R}$ is the change in electrical resistance, $\mathrm{R}$ is the initial resistance, $\Delta \mathrm{R} / \mathrm{R}$ is the fractional change in resistance and $\varepsilon$ is the flexural or compressive strain

\section{Results and discussion}

Continuous carbon fibre. The fractional change in resistance $(\Delta R / R)$ and average gauge factors of braided composites are provided in Table 2 . The change in electrical contact points during deformation is believed to be the main reason for the piezoresistive behaviour of braided composites. It can be noticed that the highest piezoresistive behaviour is obtained with the braided composites having less $\%$ of carbon fibre in the core and the strain sensibility decreases with increase in the carbon fibre \%. In the composites with higher amount of carbon fibres, there will be less change in electrical contacts during deformation due to more touching of fibres leading to a large number of electrical contact points throughout the composites. The gauge factors obtained with these braided composites (up to 23.4 due to strain up to $0.55 \%$ ) is higher than the composites with aligned carbon fibres studied previously mainly due to the type of fibre arrangement obtained with the braiding process. Due to influence of braiding process, the core carbon fibres were misaligned and changed their positions frequently along the length of composites. The change of electrical contact points is expected to be more with this kind of fibre arrangements than the composite systems with aligned carbon fibres.

\section{Chopped carbon fibre}

Distribution of chopped fibres in the matrix. The distribution of chopped carbon fibres $(1 \mathrm{~mm})$ in the matrix at $0.5 \%$ concentration as detected by optical microscopy at two different magnifications is shown in Fig. 2. It can be seen that the dispersion of chopped fibres is fairly uniform throughout the matrix and also shows good connections for electrical conductivity. However, dispersion becomes more difficult with the increase in chopped fibre length and concentration 
Table 2: Fractional change in resistance and average gauge factor of braided composites

\begin{tabular}{|c|c|c|c|c|c|c|c|c|c|}
\hline Cycle No. & \multicolumn{2}{|c|}{$\mathbf{1}$} & \multicolumn{2}{c|}{$\mathbf{2}$} & \multicolumn{2}{c|}{$\mathbf{3}$} & \multicolumn{2}{|c|}{$\mathbf{4}$} & \\
\hline $\begin{array}{c}\text { Composition } \\
\text { of core }\end{array}$ & $\begin{array}{c}\boldsymbol{\varepsilon} \\
(* \mathbf{1 0}\end{array}$ & $\Delta \mathbf{R} / \mathbf{R}$ & $\begin{array}{c}\boldsymbol{\varepsilon} \\
(* \mathbf{1 0}\end{array}$ & $\Delta \mathbf{R} / \mathbf{R}$ & $\begin{array}{c}\boldsymbol{\varepsilon} \\
\left(* \mathbf{1 0}^{-2}\right)\end{array}$ & $\Delta \mathbf{R} / \mathbf{R}$ & $\begin{array}{c}\boldsymbol{\varepsilon} \\
\left(* \mathbf{1 0}^{-2}\right)\end{array}$ & $\Delta \mathbf{R} / \mathbf{R}$ & $\begin{array}{c}\text { Average } \\
\mathbf{G F}\end{array}$ \\
\hline $23 / 77$ & 0,48 & 0,10 & 0,48 & 0,11 & 0,48 & 0,12 & 0,48 & 0,12 & 23.4 \\
\hline $47 / 53$ & 0,48 & 0,04 & 0,48 & 0,02 & 0,48 & 0,01 & 0,48 & 0,01 & 4.2 \\
\hline $100 / 0$ & 0,55 & 0,02 & 0,55 & 0,01 & 0,55 & 0,01 & 0,55 & 0,01 & 2.3 \\
\hline
\end{tabular}

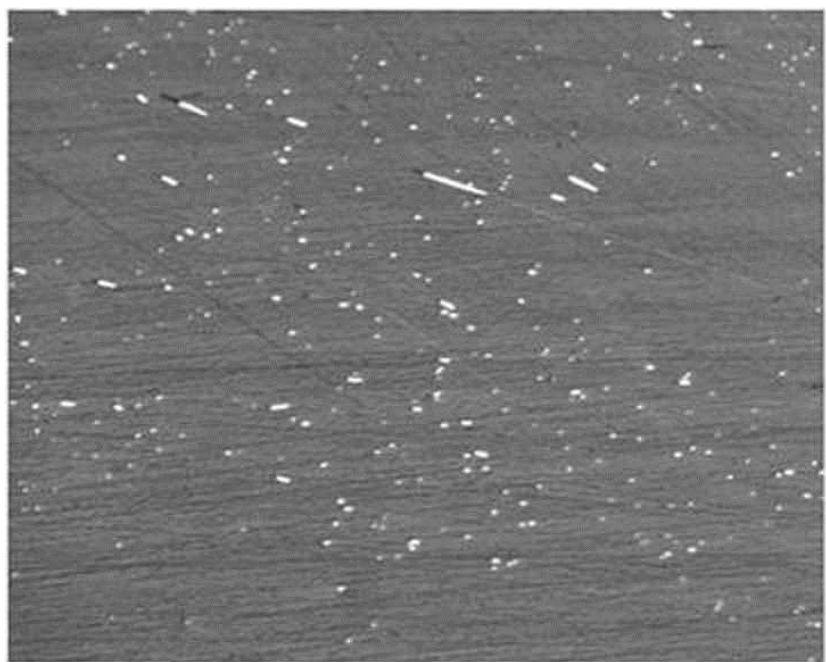

(a)

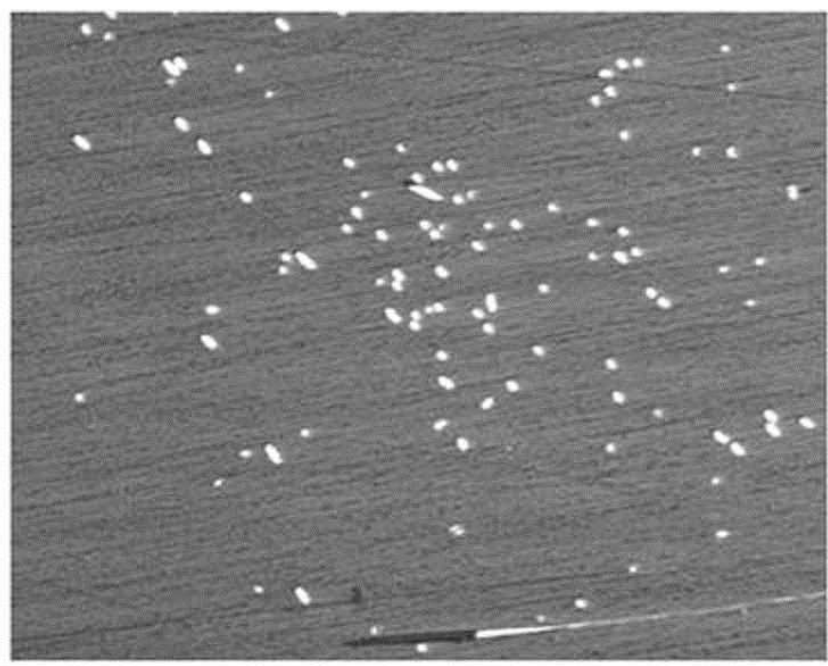

(b)

Fig. 2: Distribution of chopped fibres of $1 \mathrm{~mm}$ length within the matrix at $0.5 \%$ concentration: (a) at magnification of $10 \times 1.67$ and (b) at magnification of $20 \times 1.67$

Monotonic compression test. The results of monotonic compression test for chopped carbon fibre dispersed matrix are presented in Fig. 3. The calculated gauge factors are also indicated. It can be noticed that the samples containing $1 \mathrm{~mm}$ chopped fibre showed better strain sensitivity than 3 $\mathrm{mm}$ fibre dispersed samples in case of $0.75 \%$ and similar strain sensitivity for remaining two concentrations. Moreover, the gauge factors are higher in case of lower concentration of chopped fibres. The better strain sensitivity for lower concentrations is probably attributed to the better dispersion of chopped carbon fibres at lower concentrations. 


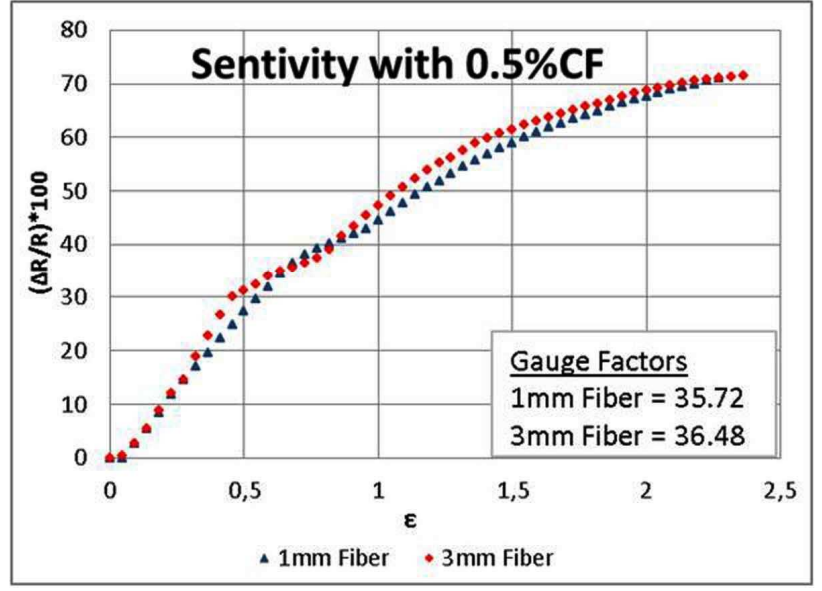

(a)

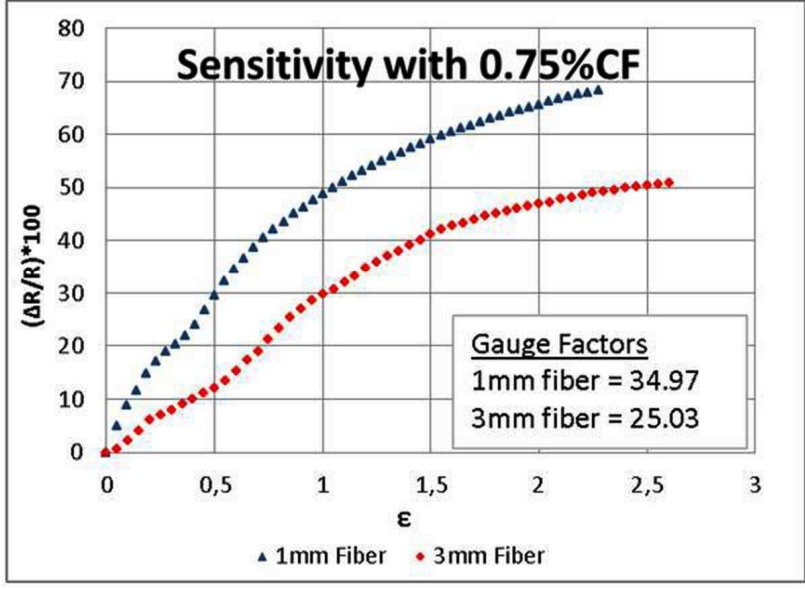

(b)

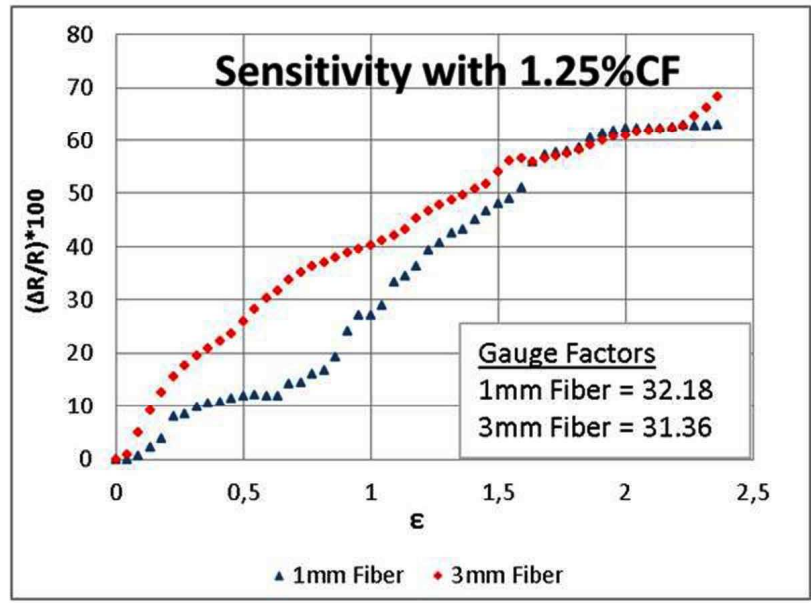

(c)

Fig 3: Variation of fractional resistivity of chopped fibres dispersed matrix with compressive strain at different concentrations: (a) $0.5 \%$, (b) $0.75 \%$ and (c) $1.25 \%$.

Cyclic compression test. The results of cyclic compression test for $3 \mathrm{~mm}$ chopped fibre dispersed samples at $1.8 \%$ strain level are provided in Table 3. The samples with $1 \mathrm{~mm}$ chopped carbon fibres showed similar sensing behaviour. It can be noticed that the average gauge factor is similar in all three concentrations studied. However, the matrix with $0.5 \%$ chopped fibre showed better strain sensitivity in the initial cycles as can be seen from the values of fractional resistance change listed in Table 3. Hence, among the three concentrations, $0.5 \%$ is more suitable for developing piezoresistive matrix since it is easier to disperse and moreover, it results in better strain sensitivity. 
Table 3: Fractional change in resistance and average gauge factors of chopped fibre dispersed matrix

\begin{tabular}{|c|c|c|c|c|c|c|c|}
\hline Cycle No. & \multicolumn{2}{|c|}{1} & \multicolumn{2}{c|}{ 2 } & \multicolumn{2}{c|}{ 3 } & \\
\hline $\begin{array}{c}\text { Conc. of } \\
\text { chopped fibre }\end{array}$ & $\boldsymbol{\varepsilon}$ & $\Delta \mathbf{R} / \mathbf{R}$ & $\boldsymbol{\varepsilon}$ & $\Delta \mathbf{R} / \mathbf{R}$ & $\boldsymbol{\varepsilon}$ & $\Delta \mathbf{R} / \mathbf{R}$ & Average GF \\
\hline $0.5 \%$ & 1.8 & 78,09 & 1.8 & 86,61 & 1.8 & 89,03 & 47 \\
\hline $0.75 \%$ & 1.8 & 67,73 & 1.8 & 83,77 & 1.8 & 88,31 & 44 \\
\hline $1.25 \%$ & 1.8 & 73,83 & 1.8 & 84,83 & 1.8 & 88,47 & 46 \\
\hline
\end{tabular}

\section{Summary}

In the present research, core reinforced hybrid carbon fibre/glass fibre braided composites have been developed for continuous monitoring of structural health. Among the various compositions studied, the braided composites with lowest amount of carbon fibre showed the best strain sensitivity. Gauge factors up to 23.4 were achieved at very low level of cyclic flexural strain $(0.55 \%)$. Moreover, it was found possible to develop a piezoresistive matrix for these braided composites by dispersing very short chopped carbon fibres (less than $3 \mathrm{~mm}$ ) at very low concentrations $(0.5 \mathrm{wt} . \%)$. Therefore, the future attempts will be made to investigate the strain sensing behaviour of the core reinforced braided composites containing chopped carbon fibre dispersed matrix in order to develop continuous monitoring system with very good sensing capability.

\section{References}

[1] N. Muto, Y. Arai, S.G. Shin, H. Matsubara, H. Yanagida, M. Sugita, T. Nakatsuji, Hybrid composites with self-diagnosing function for preventing fatal fracture, Compos. Sci. Technol. 61 (2001) 875-883.

[2] C.E. Bakis, A. Nanni, J.A. Terosky, S.W. Koehler, Self-monitoring, pseudo-ductile, hybrid FRP reinforcement rods for concrete applications, Compos. Sci. Technol. 61 (2001) 815-823.

[3] Y. Okuhara, H. Matsubara, Memorizing maximum strain in carbon-fiber-reinforced plastic composites by measuring electrical resistance under pre-tensile load, Compos. Sci. Technol. 65 (2006) 2148-2155.

[4] M. Nofar, S.V. Hoa, M.D Pugh, Failure detection and monitoring in polymer matrix composites subjected to static and dynamic loads using carbon nanotube networks, Compos. Sci. Technol. 69 (2009) 1599-1606. 